MACKINTOSH, N. J. Selective attention in animal discrimination learning. Psychological Bulletin, 1965, 64, 124-150.

SIEGEL, P. S. The role of absolute initial response strength in simple trial-anderror learning. Journal of Experimental Psychology, 1945, 35, 199-205.

SUTHERLAND, $N$. S. The learning of discriminations by animals. Endeavour, 1964, 23, 148-152.

\title{
Within-session behavioral contrast? ${ }^{1}$
}

ROBERT S. BEECROFT, University of North Dakota, Grand Forks, N. Dak. 58201

No within-session contrast was found with a mult FREXT schedule that produced positive behavioral contrast between sessions. The absence of within-session contrast agrees with an earlier study by Reynolds (1968) and poses a difficulty for response-rate interpretations of behavioral contrast.

Positive behavioral contrast refers to increased responding in one component of a multiple schedule when responding decreases in the other component. Since Reynolds's (1961) initial analysis, behavioral contrast has been studied as a between-sessions phenomenon. Recently, Reynolds (1968) sought evidence of within-session contrast during the early phases of mult VI EXT and mult VR EXT training. He reasoned, "If the prevailing rate of responding during extinction determines the magnitude of behavioral contrast, there should be a substantial negative correlation: the lower the rate of responding in extinction in the presence of $\mathrm{S}-$, the higher the rate of responding in the presence of St [p. 321]." The technique employed was to inspect the scatterplots of response rate for each 3-min St period plotted against the response rate for the immediately preceding 3-min S- period. No negative correlations were evident for either of two pigeons in either of the multiple schedules investigated, although both birds showed positive contrast. The present paper examines comparable data for rats trained with a mult FR EXT schedule that produces excellent behavioral contrast.

\section{METHOD}

A Scientific Prototype Model A-102 box was modified for discrimination training. Three male hooded rats, 78-91 days old, were trained. They had been on 23-h food deprivation for a week. Each rat was given 1 day of CRF and 1 day of FR3 training (approximately 200 45-mg Noyes pellets each day) in the presence of St, a white jewel light located above the lever. On the lst day of discrimination training, the light was on when the animal was placed in the box and continued in this state until 20 reinforcements were obtained on the prevailing FR3 schedule. The light was then turned off for a $2-\mathrm{min} \mathrm{S}$ - period. The first $S+$ period following the introduction of $\mathrm{S}$ - was extended beyond $2 \mathrm{~min}$ if necessary to insure reinforced responding

Table 1

Responses Per Minute and WithinSession Correlations

\begin{tabular}{lccrc}
\hline Rat & Day & S+ & \multicolumn{1}{c}{ S- } & r \\
\hline BC & 1 & 16.5 & 23.5 & +.39 \\
& 2 & 20.2 & 12.8 & +.04 \\
& 3 & 22.3 & 10.7 & +.24 \\
& 4 & 23.0 & 7.3 & +.68 \\
BD & 1 & 10.8 & 14.6 & +.77 \\
& 2 & 14.1 & 9.5 & -.52 \\
& 3 & 17.8 & 6.4 & +.48 \\
& 4 & 25.6 & 4.2 & +.30 \\
CB & 1 & 15.3 & 20.6 & +.01 \\
& 2 & 18.5 & 10.5 & +.16 \\
& 3 & 25.1 & 10.0 & -.29 \\
& 4 & 28.0 & 8.3 & +.16 \\
\hline
\end{tabular}

in S+. Each session began and ended with an St period. There were $11 \mathrm{~S}+$ and $10 \mathrm{~S}-$ periods daily, each 2 min in length except as noted above. Response-rate calculations do not include the first St period of the day. There were 4 days of training with mult FR3 EXT.

RESULTS AND DISCUSSION

Table 1 shows that responding increased in $\mathbf{S t}$ and decreased in $\mathbf{S}$-. The rapid development of discrimination is typical with mult FR3 EXT. It is worth noting that the response rate in $S-$ was higher than the response rate in S+ on the 1st day, in spite of the fact that the prior CRF and FR3 training was carried out in the presence of St. This initial rate bias favoring $S-$ is characteristic of the first discrimination session with rats and has been reported in other studies (e.g., Smith \& Hoy, 1954).

Having established that all three rats exhibited positive behavioral contrast, the relationship of $\mathrm{S}+$ responding to $\mathrm{S}-$ responding in the immediately preceding period was analyzed. Scatterplots were made of the pairs of response-rate scores for $\mathrm{S}-$ and $\mathrm{S}+$ periods. Pearson rs, corresponding to the scatterplots, are shown in the table. Only two of the correlations were negative. The only one to approach statistical significance was for Rat BD on Day 2. On the 3 other days for this animal, responding in $\mathrm{S}+$ was positively correlated with responding in $\mathrm{S}-$. However, a negative correlation does not necessarily imply a sequential divergence of the $\mathrm{S}+$ and $\mathrm{S}$ - period scores within a session, the form that a within-session contrast should take, and there was no sequential divergence associated with either of the negative correlations observed in this study.

The absence of within-session behavioral contrast is in complete agreement with Reynolds (1968). It may be concluded that behavioral contrast can and does occur between sessions without within-session contrast. As Reynolds has said, such data "... make it difficult to account for the development and magnitude of contrast ... by appealing directly to the prevailing rate of responding in the presence of S[p. 322]."

\section{REFERENCES}

REYNOLDS, G. S. Behavioral contrast. Journal of the Experimental Analysis of Behavior, $1961,4,57-71$.

REYNOLDS, G. S. Positive behavioral contrast: Development within sessions. Psychonomic Science, 1968, 10, 321-322.

SMITH, M. C., JR., \& HOY, W. J. Rate of response during operant discrimination. Journal of Experimental Psychology, 1954, $48,259-264$.

\section{NOTE}

1. This research was aided by a Faculty Research Grant. 